\title{
Narrowband Wavelength Selective Thermal Emitters by Confined Tamm Plasmon Polaritons
}

\author{
Zih-Ying Yang, ${ }^{1}$ Satoshi Ishii, $2,{ }^{*}$ Takahiro Yokoyama, ${ }^{2}$ Thang Duy Dao, ${ }^{2}$ Mao-Guo Sun, ${ }^{3}$ Pavel S. \\ Pankin, ${ }^{4}$ Ivan V. Timofeev, ${ }^{5,6}$ Tadaaki Nagao, ${ }^{2, *}$ and Kuo-Ping Chen ${ }^{3, *}$ \\ ${ }^{1}$ Institute of Lighting and Energy Photonics, National Chiao Tung University, 301 Gaofa 3rd Road, Tainan 711, Taiwan \\ ${ }^{2}$ International Center for Materials Nanoarchitectonics, National Institute for Materials Science (NIMS), 1-1 Namiki, Tsukuba, Ibaraki 305-0044, \\ Japan \\ ${ }^{3}$ Institute of Imaging and Biomedical Photonics, National Chiao Tung University, 301 Gaofa 3rd Road, Tainan 711, Taiwan \\ ${ }^{4}$ Institute of Engineering Physics and Radio Electronics, Siberian Federal University, Krasnoyarsk 660041, Russia \\ ${ }^{5}$ Kirensky Institute of Physics, Federal Research Center KSC SB RAS, Krasnoyarsk 660036, Russia \\ ${ }^{6}$ Laboratory for Nonlinear Optics and Spectroscopy, Siberian Federal University, Krasnoyarsk 660041, Russia
}

\section{Abstract}

Infrared wavelength selective thermal emission based on Tamm plasmon polaritons (TPPs) is experimentally demonstrated. Unlike conventional TPP structures having a thin metal layer on a DBR, the proposed structure has a thick metal under a DBR which is more robust for thermal radiation. The number of DBR pairs is a critical factor to maximize the narrowband emission: It has to satisfy the impedance matching condition, which varies with the choice of metal film. The proposed structure can achieve twice higher Q-factor for the measured emissivity compared to typical plasmonic thermal emitters. The structure is one dimensional, only consists of multilayers, and free from nano-patterning, offering a practical design in applications such as gas sensing, narrowband IR sources and in thermophotovoltaics.

TOC

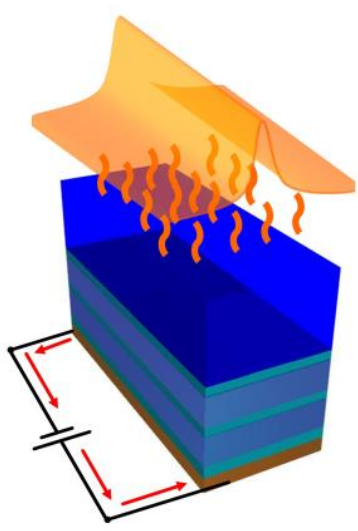




\section{Introduction}

Wavelength selective thermal emitters have gathered great attention recently due to the narrow band radiation [1-3]. By choosing specific natural materials that absorb the required wavelength region can be a way to reduce the unwanted losses, but the absorbing region and the bandwidth are limited by the intrinsic material property without adjustability [4]. In contrast, artificial nanostructures can make a tunable absorber/emitter with a narrow emission peak [5-7]. Different types of nanostructures have been investigated including photonic crystals (PCs) which utilize band edge resonance $[8,9]$, surface plasmon polaritons (SPPs) resulted from metallic grating structures [10], gap-plasmon-guided mode of asymmetric T-shape structures [11], and magnetic dipole resonance produced by metal-insulator-metal (MIM) metamaterials [12-16]. Regardless of the designs, all the structures listed above require a 2D nanofabrication process which are costly and hinder practical applications [17].

In order to achieve large area fabrications without complexity, a planar multilayer structure based on surface state resonances have huge advantage over other 2D or 3D nanostructures. Among multilayer structures, Fabry-Pérot structures have been demonstrated as wavelength selective emitters $[18,19]$. However, since they do not have stop bands, there would be multiple emission peaks thus the $\mathrm{S} / \mathrm{N}$ ratio cannot be high. On the other hand, a metallic film combined with a distributed Bragg reflector (DBR) serving as a 1D photonic crystal can be a narrow band emitter having low background level. In such a structure, Tamm plasmon polaritons (TPPs), which has a zero in-plane wavevector, has gathered great attention lately [20,21]. Since both TE and TM waves can excite TPPs, TPP structures are polarization independent. The tunability of TPPs can be readily achieved by changing the stop band of the DBR [22]. So far, TPPs have been studied from various directions, such as theoretical studies on eigenmodes [23], combination with metamaterials [24], propagating and non-propagating TPPs [25], luminescence enhancement [26], and selective thermal emitters [27]. However, little study has been done focusing on optimizing the absorption and thermal emission peak in mid-IR region based on TPP structures.

In order to reach the high quality factor (Q-factor) TPP, one way is selecting appropriate metallic materials, which has been discussed in Ref. [22]. In addition, the configuration (see Figure 1) is also an important factor for optimization. Klimov et al compared metal-side TPP structures (incident light from the thin metal film) to DBR-side TPP structures (incident light from the DBR) at visible region [28]. For metal-side TPP, metal thickness needs to be optimized to reach a perfect absorption with high Q-factor. However, for DBR-side TPP structures, the number of the DBR pairs determines the magnitude of the absorptivity rather than metal thickness [29]. That means DBR-side TPP structures can have thick metal film which behave as a good reflector but strong evanescent fields are excited in the DBR. Moreover, it can be inferred that DBR-side TPP structures can possess better mechanical stability and refractory properties (thick metal film) and lower the fabrication difficulty as the fabrication of a continuous ultra-thin film is challenging.

In this study, DBR-side TPP structures were chosen to demonstrate the ultra-sharp emission peak in mid-IR region. By designing the coupling between localized modes and the incident wave, we analytically and experimentally demonstrate enhanced absorptivity (i.e. emissivity) by DBR-side TPP structures at the designed wavelength. First, the difference between metal-side TPP structures and DBR-side TPP structures as well as the design considerations for each structure are discussed based on coupled mode theory. Then, by taking the optical constants and the impedance matching into account, the optimized conditions of DBR with different metals are presented. The best Q-factor of 36.5 is achieved in experiment by choosing $\mathrm{Al}$ as a metal film. The tunability of the DBR-side TPP structures and emission properties with different metals were experimentally demonstrated. In addition, the threshold temperature of DBR-side TPP structures using different metals were also experimentally demonstrated. 

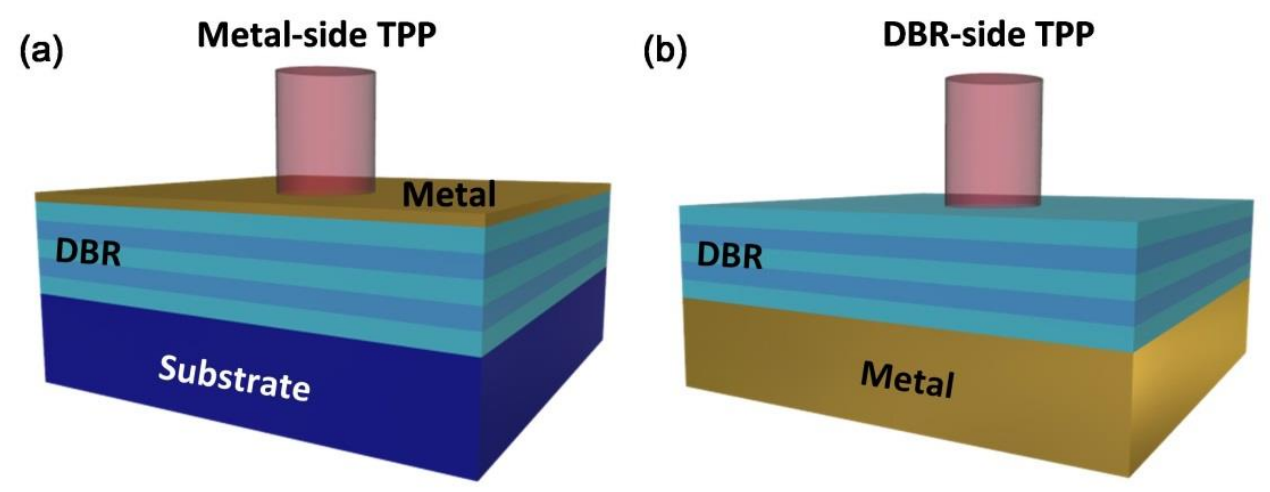

Figure 1 Schematic diagrams of (a) metal-side TPP structure with a thin metal film where light is illuminated from thin metal layer; (b) DBR-side TPP structure where light is illuminated from DBR side with an opaque metal film below.

In Figure 2, two kinds of TPP structures having the same $4 \mu \mathrm{m}$ resonance are compared. One is the metal-side TPP structure with a thin metal film (Fig. 2(a)), while the other is the DBR-side TPP structure with an opaque metal film (Fig. 2(b)). As examples, two pairs of DBR composed of $\mathrm{Si}$ and $\mathrm{SiO}_{2}$ layers, combined with an additional Si layer adjacent to an Al metal film were chosen to form the TPP structures. As shown in Figs. 2(b) and 2(e), the 2D magnetic field distributions clearly show the localized enhancement at the interfaces of the DBR and metal, and then, the fields exponentially decay into both the metal side and the DBR side. The field enhancement of DBR-side TPP structure is $\sim 20$ times larger than that of metal-side TPP structure. The simulated reflectance of the metal-side and the DBR-side TPPs aimed at $4 \mu \mathrm{m}$ are shown in Figs. 2(c) and 2(f). The DBR-side TPP provides a 10 times narrower bandwidth and a much higher absorptance compared to the metal-side TPP.

In order to understand the confined energy in TPP, the relaxation time $(\tau)$ can be used to define the ratio of the stored energy $(E)$ and the leakage power $(P)$ as,

$$
\frac{\tau}{2}=\frac{E}{P}
$$

The temporal coupled-mode theory can describe the Q-factor of TPP structures by considering the relaxation time [30, $31]$

$$
\begin{aligned}
& \frac{1}{\tau}=\frac{1}{\tau_{D B R}}+\frac{1}{\tau_{M e t a l}}+\frac{1}{\tau_{A}}, \\
& Q=\frac{\tau \varpi_{T P P}}{2},
\end{aligned}
$$

where $\tau_{D B R}, \tau_{\text {Metal }}$, and $\tau_{A}$ are the relaxation times due to the transmission loss of the DBR mirror, the transmission and absorption loss of the metals, respectively, $\varpi_{T P P}$ is the TPP resonance frequency.

The minimization of the three losses is required for better energy storage (larger $\tau$ ). The critical coupling condition for DBR-side TPP occurs at larger relaxation time than metal-side TPP (see Fig. S1 in supplementary material). Therefore, a DBR-side TPP structure can support a resonance with a high Q-factor due to lower power of leakage. 

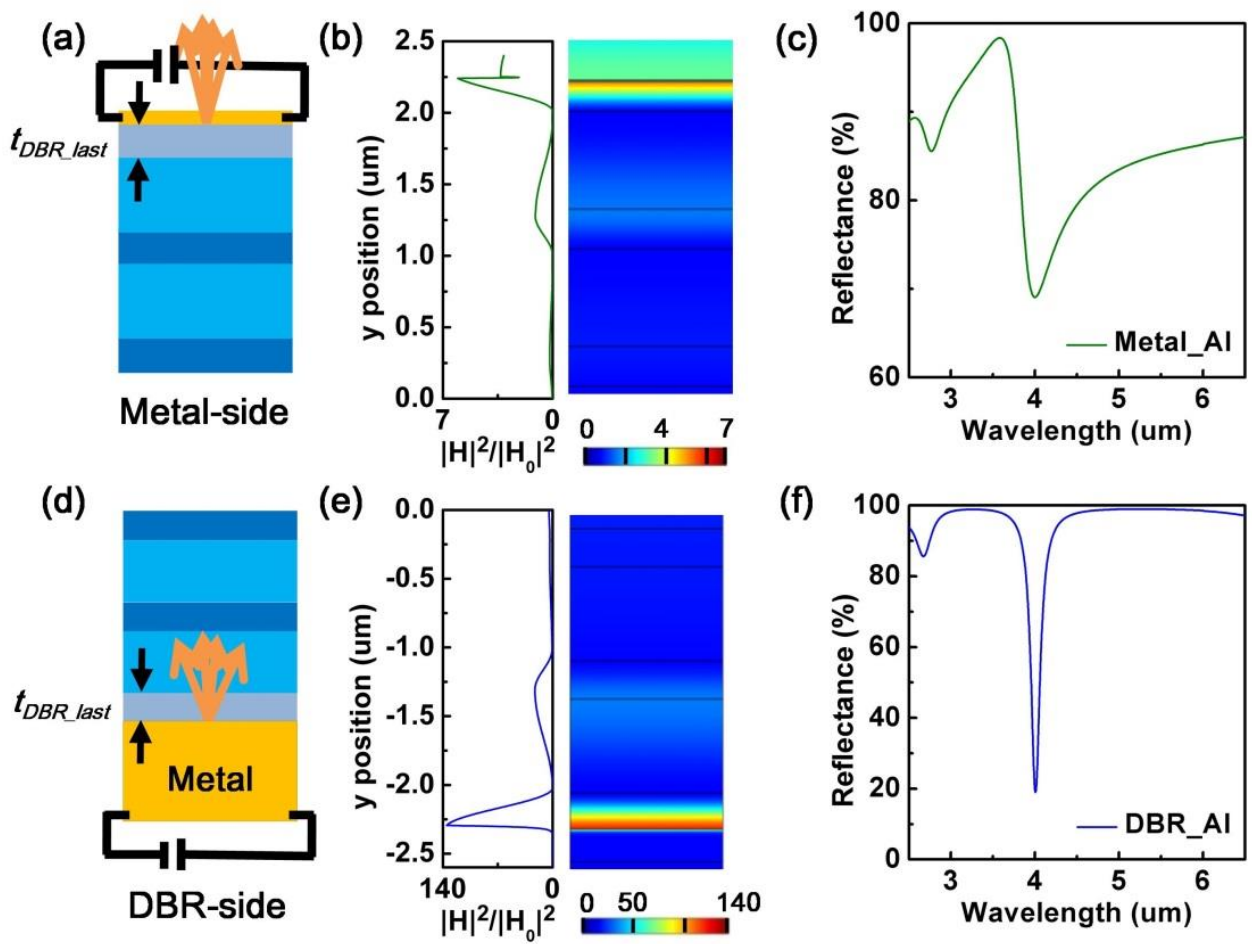

Figure 2 Comparison of the metal-side $(\mathrm{Al}=10 \mathrm{~nm}|\mathrm{Si}=220 \mathrm{~nm}| 2$ pairs DBR aimed at $4 \mu \mathrm{m})$ and DBR-side TPP structures (2 pairs DBR aimed at $4 \mu \mathrm{m}|\mathrm{Si}=273 \mathrm{~nm}| \mathrm{Al}=250 \mathrm{~nm}$ ). (a, d) schematic diagram, (b, e) cross-section plot along $\mathrm{z}$ axis and normalized magnetic field intensity (c, f) simulated reflectance spectra for the two TPP structures aimed at $4 \mu \mathrm{m}$. In the emission experiment, current was applied to the metal layer (shown as the circuit with a voltage source) to heat the sample, and the yellow arrows represent the thermal emission from the interface of the DBR/metal due to TPPs resonance.

To investigate the design consideration of the two TPP structures, aluminum (Al) and gold (Au) are chosen as the absorbing/emitting layer to calculate the absorptance spectra maps with various number of the DBR pairs as well as various thicknesses of the metal, as shown in Fig. 3. When increasing the number of DBR pairs, the absorptance will slightly increase till six pairs DBR for metal-side TPP structures (Figs. 3(a) and 3(e)), however, there is an optimized number of the DBR pairs for DBR-side TPP structures (Figs. 3(c) and 3(g)). In terms of the metal film thickness, larger TPP absorptance is realized by decreasing the thickness for metal-side TPP structure (Figs. 3(b) and 3(f)) whereas the DBR-side TPP structure has the opposite (Figs. 3(d) and 3(h)). Note that when aiming at mid-IR region, the magnitude of $\varepsilon^{\prime}$ of the metal is much higher than that in visible/near-IR region, which makes the metal's thickness to be only several nanometers to allow photon penetration through the metal film, as can be seen in Figs. 3(b) and 3(f). Because of the difficulty in getting smooth and contentious ultrathin metal layer, metal-side TPP structures are disadvantageous in fabrication. To sum up, for DBR-side TPP structures, an optimized number of the DBR pairs combined with a thick metal layer is necessary for strong TPP coupling. 

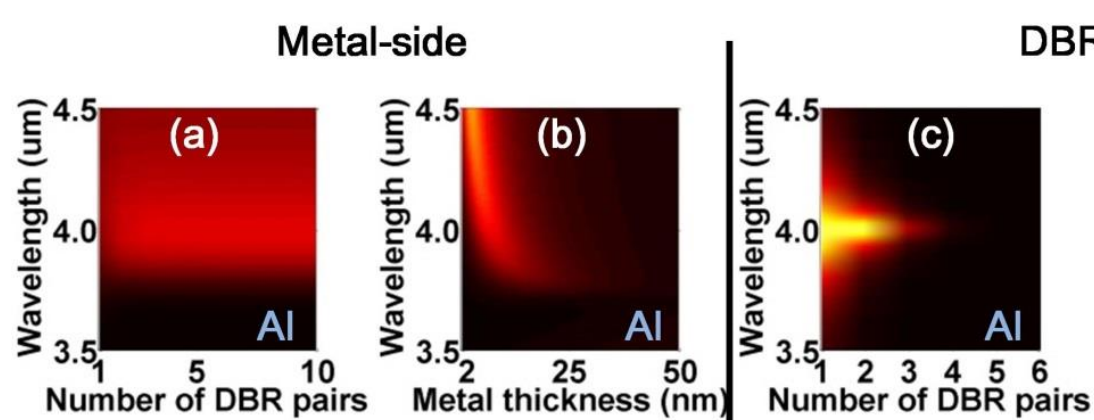

DBR-side
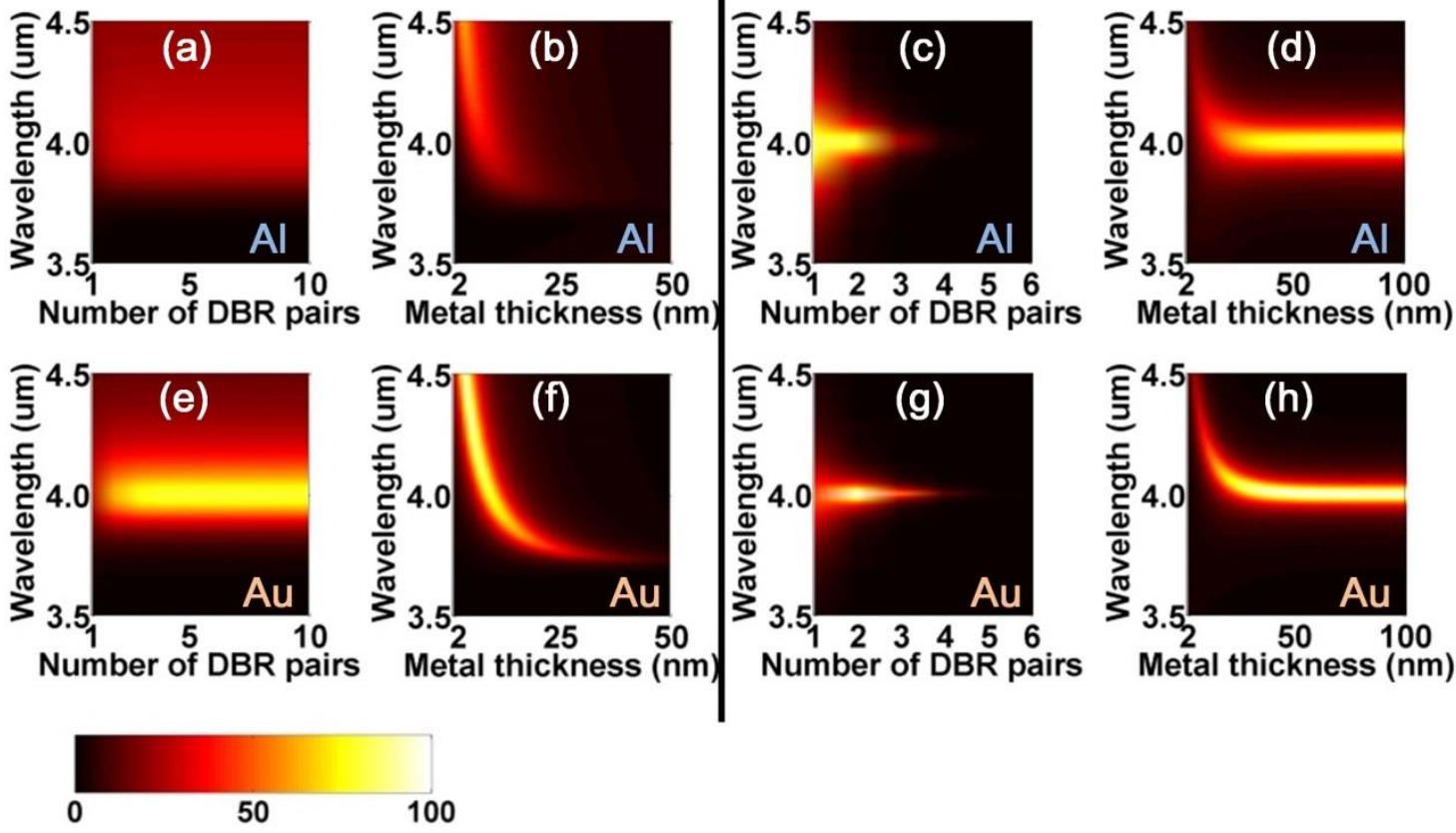

Figure 3 Simulated 2D absorptance maps of the TPP structures. Using Al as the emitting layer: for metal-side TPP design (a) the number of the DBR pairs is varied from 1 pair to 10 pairs, and (b) the metal layer thickness is varied from $2 \mathrm{~nm}$ to $50 \mathrm{~nm}$; for DBR-side TPP design (c) the number of the DBR pairs is varied from 1 pair to 6 pairs, and (d) the metal layer thickness is varied from $2 \mathrm{~nm}$ to $100 \mathrm{~nm}$. Using Au as the emitting layer: for metal-side TPP design (e) the number of the DBR pairs is varied from 1 pair to 10 pairs, and (f) the metal layer thickness is varied from $2 \mathrm{~nm}$ to 50 $\mathrm{nm}$; for DBR-side TPP design ( $\mathrm{g}$ ) the number of the DBR pairs is varied from 1 pair to 6 pairs, and (h) the metal layer thickness is varied from $2 \mathrm{~nm}$ to $100 \mathrm{~nm}$. The thickness of the $\mathrm{Si} / \mathrm{SiO}_{2}$ forming DBR is equal to a quarter of optical path length of $4 \mu \mathrm{m}$ which is the targeted resonance wavelength.

As can be seen from Figure 3, the choice of metal is a key factor in TPP resonance. To further investigate how the selection of metals and the number of the DBR pairs affect the TPP resonance of DBR-side devices, two coinage metals, $\mathrm{Au}, \mathrm{Al}$, and two refractory metals, molybdenum (Mo), tungsten (W), were chosen. The dielectric functions of $\mathrm{Al}, \mathrm{Au}$, Mo, and W are shown in Fig. 4(a). The optical constants of Al, Mo and W were characterized by spectroscopic ellipsometry (SENDIRA, Sentech). The optical constant of Au was taken from the literature [32]. Figures 4(b)-4(e) show the simulated absorptance spectra for DBR-side TPP structures aimed at $\sim 4 \mu \mathrm{m}$ with increasing number of the DBR pairs. When increasing the number of the DBR pairs from one to four, the absorptance decreases from $\sim 80 \%$ to $4 \%$ for Al samples but the Q-factor is the highest when the number of DBR pair is two. In Fig. 4(b), for Au samples, the optimized number of the DBR pair is also two. However, different from $\mathrm{Al}$ and $\mathrm{Au}$, the absorptance is suppressed as the number of the DBR pair increases from one to four for Mo and W samples, as can be seen in Figs. 4(d) and 4(e). The reason behind is that the coupling efficiency of TPP depends on the optical impedance matching (i.e. minimization in the reflection) at the DBR/metal interface. Since the $\mathrm{Al}$ and $\mathrm{Au}$ have higher reflection coefficients (i.e. larger negative $\left.\varepsilon^{\prime}\right)$ than Mo and $\mathrm{W}$, using $\mathrm{Al}$ or Au can support a stronger TPP resonance. In addition, $\mathrm{Au}$ or $\mathrm{W}$ can provide a narrower bandwidth than $\mathrm{Al}$ or Mo because the $\varepsilon$ " of the former metals are smaller. Consequently, there are optimized numbers for the DBR pairs when using different metals due to the impedance matching. The metal with larger negative $\varepsilon^{\prime}$ requires DBRs with higher reflectance which leads to a narrower resonance, such as $\mathrm{Al}$ and $\mathrm{Au}$. It is worth noting that the metal-side TPP using Au (Figs. 3(e) and 3(f)) can support a stronger TPP than using Al (Figs. 3(a) and 3(b). However, the performances of the DBR-side structures with the Al and Au are comparable (Figs. 3 (c), (d), (g) and (h)). This means that the optical constant of a metal gives more restrictions to the metal-side TPP structures than the DBRside TPP structures. 
For DBR-side TPP, the critical coupling condition is $\tau_{D B R}=\tau_{A}$. Based on Eq. (3), the Q-factor is proportional to the relaxation time as,

$$
Q \propto \tau \propto \tau_{A}
$$

Since the stored energy is the same, the relaxation time has a proportional relationship to the reflectance/absorptance as [see the supplementary material]:

$$
\tau_{D B R}: \tau_{A}=\frac{1}{T_{D B R}}: \frac{1}{A_{\text {Metal }}},
$$

where the $T_{D B R}$ and $A_{\text {Metal }}$ are the transmittance of the DBR mirror and absorptance of the metal, respectively.

In addition, at normal incidence, when defining the reflectivity by the refractive index $n$ and extinction coefficient $k$ of metal [33], the Q-factor for DBR-side TPP can be simplified as,

$$
Q \propto \frac{1}{A_{\text {Metal }}}=\frac{1}{1-R_{\text {Metal }}} \propto \frac{k^{2}}{n} .
$$

Thus, considering $n^{2}<<k^{2}$ for metals, the Q-factor of DBR-side TPP can be approximated as $k^{2} / n$. As shown in Fig. 4, the Q-factor of $\mathrm{Au}$ is the highest, then decreases as $\mathrm{Al}, \mathrm{W}$, finally the Mo. The trend fits well to $k^{2} / n$ values for each metal at $4 \mu \mathrm{m}\left(k^{2} / n\right.$ values for $\mathrm{Au}, \mathrm{Al}, \mathrm{W}$, and Mo are 391, 101, 58 and 39).
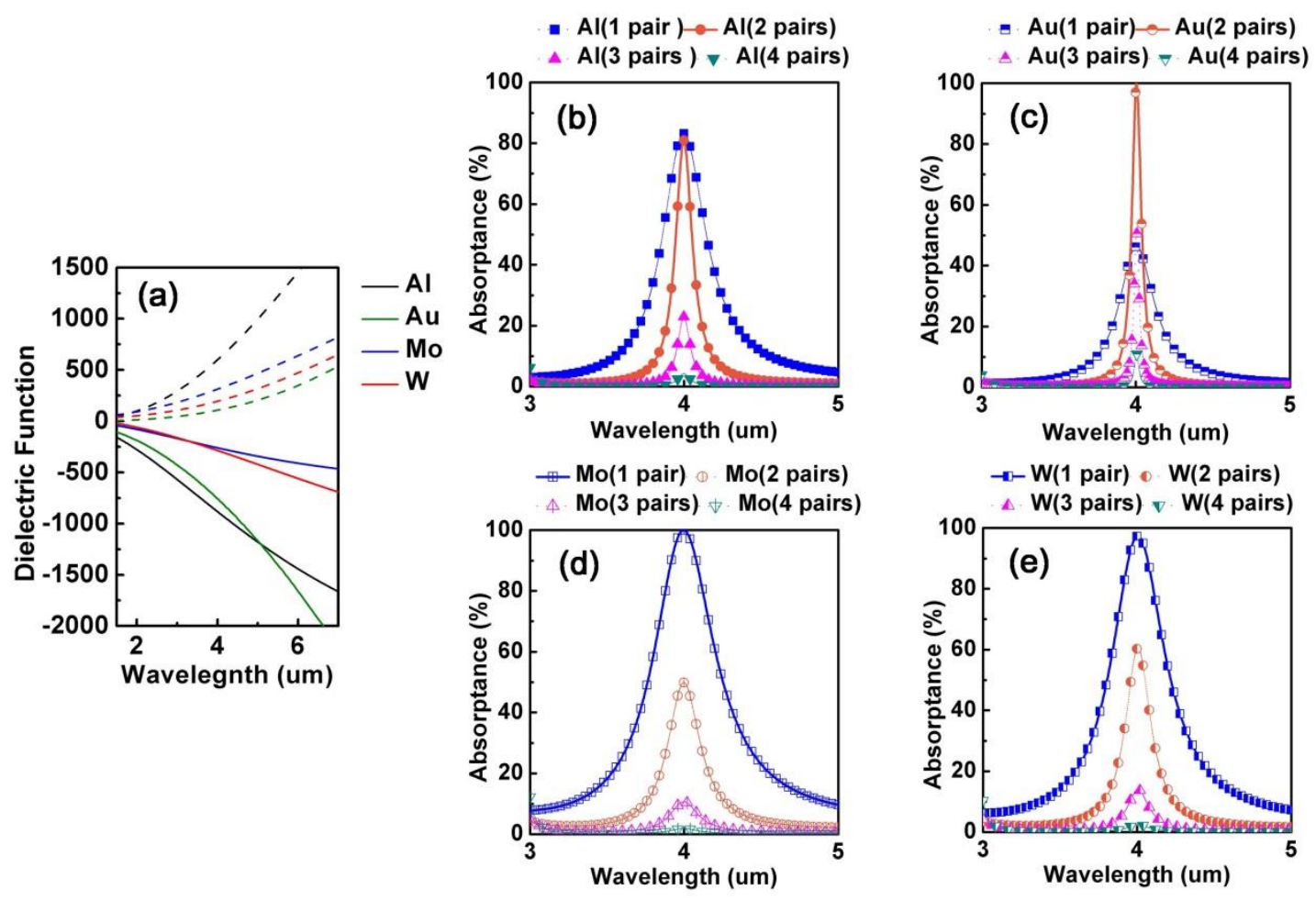

Figure 4 (a) Complex dielectric functions of $\mathrm{Al}, \mathrm{Au}, \mathrm{Mo}$, and $\mathrm{W}$. The DBR-side TPP structures aimed at $4 \mu \mathrm{m}$ with the number of the DBR pairs from 1 to 4, using (b) Al, (c) Au, (d) Mo, and (e) W as the emitting metal layer with the fixed $t_{D B R \_l a s t}=273 \mathrm{~nm}, 265 \mathrm{~nm}, 263 \mathrm{~nm}$, and $260 \mathrm{~nm}$, respectively . 


\section{Experiments and discussions}

In experiment, samples aiming at mid-IR were fabricated for $\mathrm{Au}, \mathrm{Al}, \mathrm{Mo}$, and $\mathrm{W}$. The DBR is composed of $\mathrm{Si}$ and $\mathrm{SiO}_{2}$ because it can provide a large photonic bandgap which can achieve high reflectance by only two pairs of $\mathrm{Si} / \mathrm{SiO}_{2}$ [27]. The thickness of the two-pair DBR using $\mathrm{Si}$ and $\mathrm{SiO}_{2}$ are equal to the quarter optical path length of the targeted wavelength. First, two pair DBR were deposited on a sapphire substrate, then, an additional Si layers was deposited by a magnetron sputtering machine to target each emission wavelength in which the Si layers serve as $t_{D B R}$ last. Afterwards, a metal layer of $200 \mathrm{~nm}$ was deposited on the top of the DBR by an e-gun deposition instrument. Only for Au sample, an adhesive Ti layer of $5 \mathrm{~nm}$ was deposited before the Au film.

Figure 5(a) shows the SEM cross section image for $4.6 \mu \mathrm{m}$ sample with Au. Figure 5(b) shows the simulated reflectance spectra (solid line) and simulated absorptance spectra (dashed line). The simulated spectra were calculated by the transfer-matrix method. An FTIR spectrometer (iS50R, Thermo Scientific Nicolet) was used for reflectance measurement with an opaque Au film as a reference. Figure 5(c) shows the measured reflectance spectra at room temperature. The resonant positions match well to the simulation results. Clearly, by designing the layer thickness, the tunability of the DBR-side TPP structures is demonstrated in experiment. The slight discrepancy between the simulation and experimental results might be due to the adhesion layer at the interface.
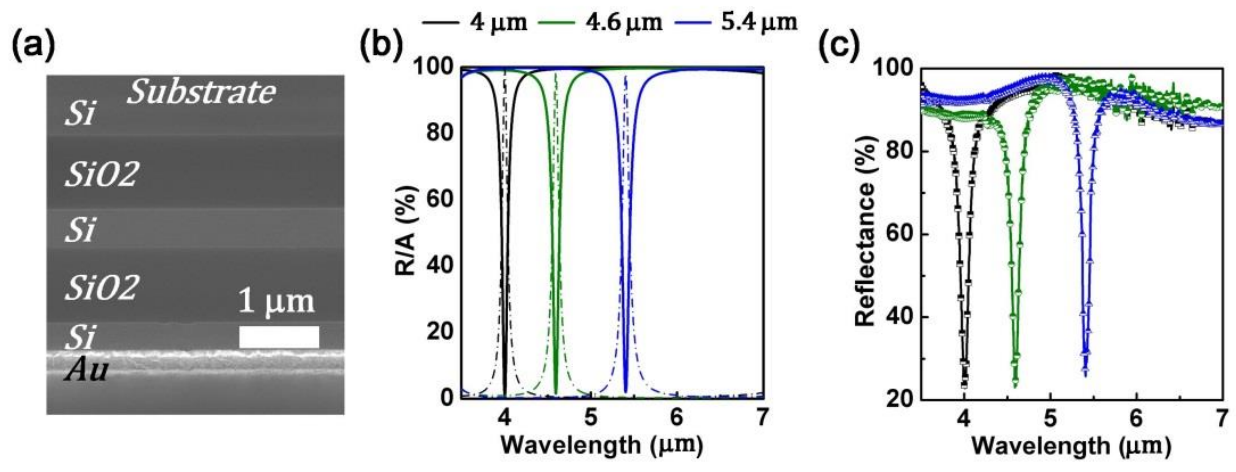

Figure 5 (a) SEM cross-sectional image of the Au sample with $\lambda_{D B R}=5 \mu \mathrm{m}$ aimed at $4.6 \mu \mathrm{m}$, the thickness for each layer is $392.2 \mathrm{~nm}(\mathrm{Si}), 765.1 \mathrm{~nm}\left(\mathrm{SiO}_{2}\right), 392.2 \mathrm{~nm}(\mathrm{Si}), 765 \mathrm{~nm}\left(\mathrm{SiO}_{2}\right), t_{D B R}$ last $=286 \mathrm{~nm}(\mathrm{Si}), \mathrm{Au}=193.7 \mathrm{~nm}$ with Ti $=5 \mathrm{~nm}$ as the adhesion layer. (b) Simulated reflectance (solid line) and absorbance (dashed line) spectra, and (c) measured reflectance spectra for DBR-side TPP structures with $\mathrm{Si} / \mathrm{SiO}_{2} \mathrm{DBR}$ and a $200 \mathrm{~nm} \mathrm{Au}$ film aimed at $4 \mu \mathrm{m}, 4.6 \mu \mathrm{m}$, and $5.4 \mu \mathrm{m}$.

In the thermal emission measurement, the sample with a thermocouple mounted on the backside was put into an ultrahigh vacuum system $\left(10^{-8}\right.$ to $10^{-9}$ Torr $)$ and heated by applying DC current to the metal layer. The sample sizes were 5 $\times 25 \mathrm{~mm}^{2}$. The emission spectra of a blackbody paint sample were used as a reference. After heating up the sample, the thermal emission was collected by an FTIR spectrometer. Because the bottom metal layer is opaque for DBR-side TPP structures, the impedance matching (i.e. number of the DBR pairs) will decide how much light can reach the interface. In order to study how the metals affect the impedance matching of DBR-side TPP structures, four different metal layers were tested in the DBR-side TPP structures. Figures 6(a)-6(d) show the simulated spectra for Al, Au, Mo, and W samples, respectively. In Figs. 6(e)-6(h), the measured reflectance and emissivity are shown. The measurement results match well to the simulations. As for the emissivity spectra, the measured results are slightly red-shifted compared to the simulated results. The reason might be the refractive index changes at high temperatures and crystallinity change due to the heating during the thermal emission measurement. The estimated temperatures for the $\mathrm{Al}$ and Au samples are $\sim 100 \pm 20{ }^{\circ} \mathrm{C}$, and $\sim 400 \pm 50{ }^{\circ} \mathrm{C}$ for the Mo and $\mathrm{W}$ samples during the emission experiments. The measured emissivities can be also compared to the results in Fig. 4. As can be seen in Fig. 4(a), the $\varepsilon^{\prime}$ around $4.2 \mu \mathrm{m}$ are similar between $\mathrm{Al}$ and $\mathrm{Au}$, and more negative compared to $\mathrm{Mo}$ and $\mathrm{W}$, which results in higher emissivity (i.e. absorptance) for $\mathrm{Al}$ and Au sample than the Mo and W samples. 

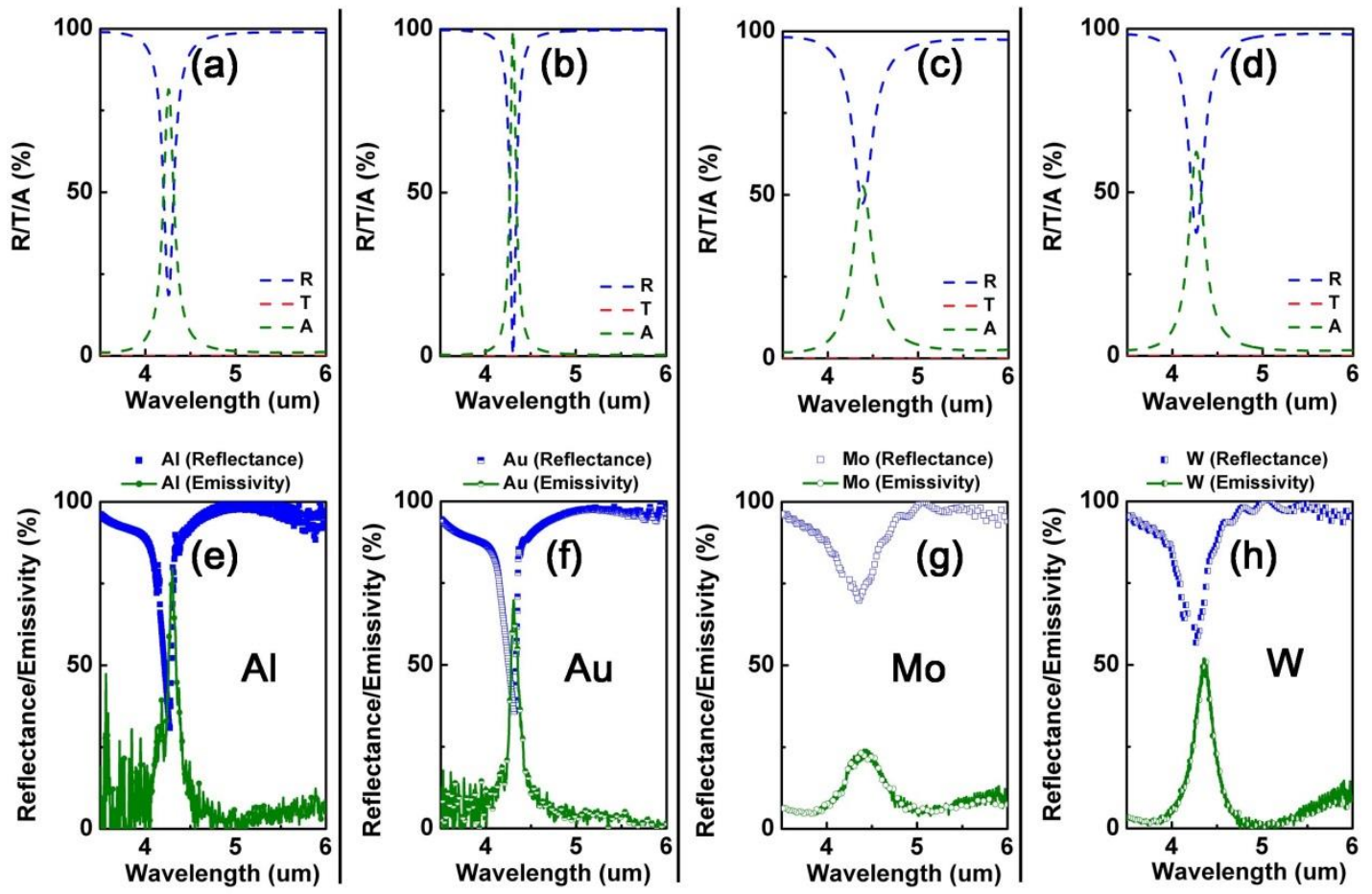

Figure 6 DBR-side TPP emitters aimed at $4.2 \mu \mathrm{m}$ : simulated reflectance, transmittance, and absorptance spectra using (a) Al, (b) Au, (c) Mo, and (d) W as the emitting layers; and the measured reflectance and emissivity for (e) Al, (f) Au, (g) Mo, and (h) W.

Further detailed studies are presented for another Al TPP structures aimed at $\sim 4 \mu \mathrm{m}$. Figure 7(b) shows the measured reflectance, which has a dip value $<0.2$, a bandwidth $=110 \mathrm{~nm}$, and the Q-factor $=36.5$. In addition, the measured emissivity spectra using heating current from $0.6 \mathrm{~A}$ to $0.85 \mathrm{~A}$ (temperature ranging from $\sim 115^{\circ} \mathrm{C}$ to $\sim 270^{\circ} \mathrm{C}$ ) was shown in Fig. 7(c). When increasing the heating current, the emissivity peak $\left(\lambda_{\text {peak }}\right)$ red-shifted by $\sim 60 \mathrm{~nm}$, and the Q-factor slightly decreased from 29.8 to 27.9. When the Q-factor of the Al TPP structure is compared to other metamaterial thermal emitters, it is superior to the Al MIM structure (Q-factor $=14$ at $4 \mu \mathrm{m}$ ) by a factor of 2.0 [17], the Au rectangular-groove structure (Q-factor $=12$ at $5.5 \mu \mathrm{m})$ by a factor of 2.3 [34] and the Au cross-shaped MIM structure (Qfactor $=9.2$ at $5.8 \mu \mathrm{m})$ by a factor of $\sim 3[13]$.

(a)

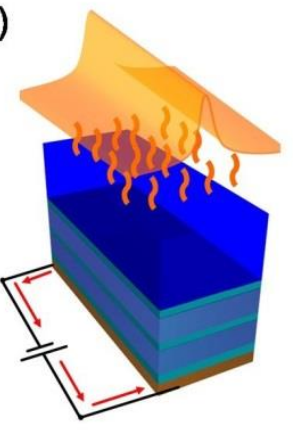

(b)

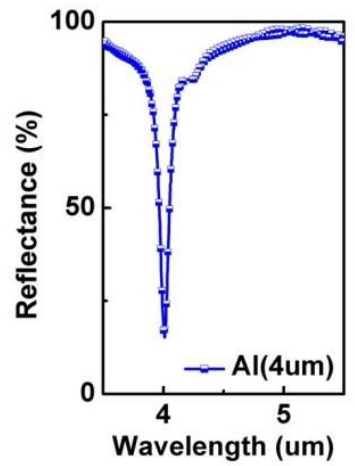

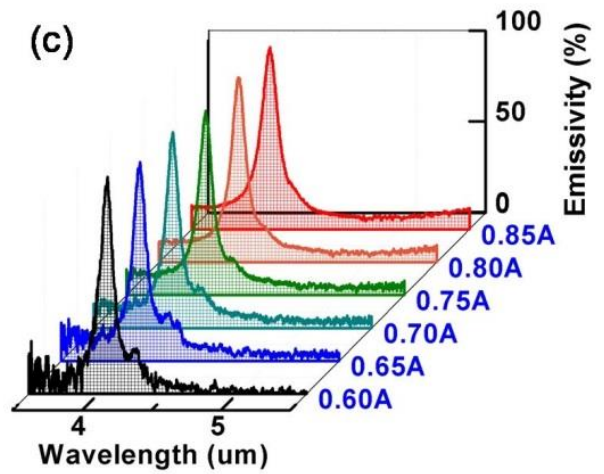

Figure $7 \mathrm{DBR}$-side TPP structure using $\mathrm{Al}$ with 2 pairs of $\mathrm{Si} / \mathrm{SiO}_{2} \mathrm{DBR}$ aimed at $4 \mu \mathrm{m}\left(t_{D B R}\right.$ last $=273 \mathrm{~nm}$, same design as shown in Fig. 4(b)): (a) schematic diagram of the TPP structure, (b) measured reflectance spectrum at room temperature, (c) emissivity spectra where the heating current varies from 0.6 A to $0.85 \mathrm{~A}$. 
Determining the operational temperature range is inevitable in practice [35]. To investigate the damage threshold of the TPP structures using different metal materials, the samples were heated by increasing the heating current (i.e. temperature) till the emission peak disappeared or shifted significantly. Table 1 lists the melting temperatures $\left(T_{m e l t i n g}\right)$, threshold heating powers $\left(P_{t h r e}\right)$, and threshold heating temperatures $\left(T_{\text {thre }}\right)$ of the fabricated samples using $\mathrm{Al}, \mathrm{Au}$, Mo, and $\mathrm{W}$ in the DBR-side TPP structures. Because of their relatively low melting temperatures and threshold heating power, $\mathrm{Al}$ and $\mathrm{Au}$ samples are suitable for operating temperatures below $300{ }^{\circ} \mathrm{C}$ (corresponds to ideal blackbody's radiation peak $\lambda_{B B}=4 \mu \mathrm{m}$ to $6 \mu \mathrm{m}$ ), while Mo and $\mathrm{W}$ samples can be used for shorter wavelength since the sample can be heated up to $\sim 800{ }^{\circ} \mathrm{C}\left(\lambda_{B B}=2.5 \mu \mathrm{m}\right.$ to $\left.4 \mu \mathrm{m}\right)$ and still retain the emission shape. It can be inferred from Table 1 that the threshold temperature is proportional to the melting temperature. Note that the threshold temperature of a thin film is much lower than the bulk material's melting temperature as listed in Table 1 . The threshold temperature of $\sim 800{ }^{\circ} \mathrm{C}$ for $\mathrm{W}$ sample is coincident with the limited stability temperature of $\mathrm{W}$ reported in [36]. If increasing the temperature up to $850^{\circ} \mathrm{C}$, the degradation occurred at the tungsten film's interface.

Table 1 The melting temperatures $\left(T_{\text {melting }} s\right)$, threshold heating powers $\left(P_{\text {thre }} s\right)$, and the threshold heating temperatures $\left(T_{\text {thre }}\right)$ of DBR-side TPP structures using Al, Au, Mo, and W.

\begin{tabular}{|c|c|c|c|}
\hline Material & $T_{\text {melting }}\left({ }^{\circ} \mathrm{C}\right)[37]$ & $P_{\text {thre }}\left(\mathrm{mW} / \mathrm{mm}^{2}\right)$ & $T_{\text {thre }}\left({ }^{\circ} \mathrm{C}\right)$ \\
\hline $\mathrm{Al}$ & 660 & 9.0 & 270 \\
\hline $\mathrm{Au}$ & 1064 & 14.5 & 360 \\
\hline $\mathrm{Mo}$ & 2623 & 34.0 & 750 \\
\hline $\mathrm{W}$ & 3422 & 34.6 & 800 \\
\hline
\end{tabular}

\section{Conclusion}

We have shown analytically and experimentally that DBR-side TPP structures can demonstrate narrowband selective absorption and thermal emissions in the mid-IR region. Conventionally studied metal-side TPP structures can only achieve broad and shallow absorption peak due to the large $\left|\varepsilon^{\prime}\right|$ of the metal in the mid-IR region. In contrast, DBR-side TPP structure can mitigate the intrinsic property of the metal, because the design allow more photons to be coupled to the TPPs resonance. Using Al or Au as a metal layer in the DBR-side TPP structures, we obtained nearly twice higher or more Q-factors than other metamaterial based thermal emitters. Besides Au and Al, the refractory Mo and $\mathrm{W}$ can be also used to obtain high emissivity (i.e. absorptivity). The lithography-free feature of the DBRside TPP structure is more practical compared to other structures having $2 \mathrm{D}$ or $3 \mathrm{D}$ nano-patterns, making our structure to be suitable in various mid-IR applications such as gas sensing, narrowband IR source, and thermophotovoltaics.

\section{References}

1. Lenert, A., et al., A nanophotonic solar thermophotovoltaic device. Nature nanotechnology, 2014. 9(2): p. 126-130.

2. $\quad$ Greffet, J.-J., et al., Coherent emission of light by thermal sources. Nature, 2002. 416(6876): p. 61-64.

3. De Zoysa, M., et al., Conversion of broadband to narrowband thermal emission through energy recycling. Nature Photonics, 2012. 6(8): p. 535-539.

4. Torsello, G., et al., The origin of highly efficient selective emission in rare-earth oxides for thermophotovoltaic applications. Nature Materials, 2004. 3(9): p. 632-637.

5. Abbas, M.N., et al., Angle and polarization independent narrow-band thermal emitter made of metallic disk on SiO2. Applied Physics Letters, 2011. 98(12): p. 121116.

6. Landy, N.I., et al., Perfect metamaterial absorber. Physical review letters, 2008. 100(20): p. 207402.

7. Inoue, T., et al., Realization of narrowband thermal emission with optical nanostructures. Optica, 2015. 2(1): p. 2735.

8. Celanovic, I., N. Jovanovic, and J. Kassakian, Two-dimensional tungsten photonic crystals as selective thermal emitters. Applied Physics Letters, 2008. 92(19): p. 193101.

9. Rinnerbauer, V., et al., High-temperature stability and selective thermal emission of polycrystalline tantalum photonic crystals. Optics express, 2013. 21(9): p. 11482-11491.

10. Ikeda, K., et al., Controlled thermal emission of polarized infrared waves from arrayed plasmon nanocavities. Applied Physics Letters, 2008. 92(2): p. 1117.

11. Liao, C.Y., et al., Quasi-coherent thermal radiation with multiple resonant plasmonic cavities. Applied Physics Letters, 2016. 109(26): p. 261101.

12. Streyer, W., et al., Selective absorbers and thermal emitters for far-infrared wavelengths. Applied Physics Letters, 2015. 107(8): p. 081105. 
13. Liu, X., et al., Taming the blackbody with infrared metamaterials as selective thermal emitters. Physical review letters, 2011. 107(4): p. 045901.

14. Cheng, C.-W., et al., Wide-angle polarization independent infrared broadband absorbers based on metallic multisized disk arrays. Optics express, 2012. 20(9): p. 10376-10381.

15. Shemelya, C., et al., Stable high temperature metamaterial emitters for thermophotovoltaic applications. Applied Physics Letters, 2014. 104(20): p. 201113.

16. Chen, H.-H., et al., Double wavelength infrared emission by localized surface plasmonic thermal emitter. Applied Physics Letters, 2014. 104(8): p. 083114.

17. Dao, T.D., et al., Infrared perfect absorbers Fabricated by colloidal mask etching of Al-Al2O3-Al trilayers. ACS Photonics, 2015. 2(7): p. 964-970.

18. Wang, Z., et al., Tunneling-enabled spectrally selective thermal emitter based on flat metallic films. Applied Physics Letters, 2015. 106(10): p. 101104.

19. Wang, L., S. Basu, and Z. Zhang, Direct measurement of thermal emission from a Fabry-Perot cavity resonator. Journal of Heat Transfer, 2012. 134(7): p. 072701.

20. Azzini, S., et al., Generation and Spatial Control of Hybrid Tamm Plasmon/Surface Plasmon Modes. ACS Photonics, 2016. 3(10): p. 1776-1781.

21. Auguié, B., et al., Tamm plasmon resonance in mesoporous multilayers: toward a sensing application. ACS Photonics, 2014. 1(9): p. 775-780.

22. Chang, C.-Y., et al., Tunability and Optimization of Coupling Efficiency in Tamm Plasmon Modes. IEEE Journal of Selected Topics in Quantum Electronics, 2015. 21(4): p. 262-267.

23. Kaliteevski, M., et al., Tamm plasmon-polaritons: Possible electromagnetic states at the interface of a metal and a dielectric Bragg mirror. Physical Review B, 2007. 76(16): p. 165415.

24. Lu, H., et al., Optical Tamm states in hetero-structures with highly dispersive planar plasmonic metamaterials. Applied Physics Letters, 2013. 102(11): p. 111909.

25. Zhang, X.-L., et al., Strongly Localized Evanescent Optical Tamm States at Metal-DBR Interface. Journal of Lightwave Technology, 2013. 31(10): p. 1654-1659.

26. Brückner, R., et al., Phase-locked coherent modes in a patterned metal-organic microcavity. Nature Photonics, 2012. 6(5): p. 322-326.

27. Yang, Z.-y., et al., Tamm plasmon selective thermal emitters. Optics Letters, 2016. 41(19): p. 4453-4456.

28. Klimov, V.V., et al., Optical Tamm state and giant asymmetry of light transmission through an array of nanoholes. Physical Review A, 2015. 92(6): p. 063842.

29. Auguié, B., A. Bruchhausen, and A. Fainstein, Critical coupling to Tamm plasmons. Journal of Optics, 2015. 17(3): p. 035003.

30. Fan, S., W. Suh, and J. Joannopoulos, Temporal coupled-mode theory for the Fano resonance in optical resonators. JOSA A, 2003. 20(3): p. 569-572.

31. Timofeev, I.V., et al., Chiral Optical Tamm States: Temporal Coupled-Mode Theory. arXiv preprint arXiv:1703.00310, 2017.

32. Olmon, R.L., et al., Optical dielectric function of gold. Physical Review B, 2012. 86(23): p. 235147.

33. Born, M. and E. Wolf, Principles of optics: electromagnetic theory of propagation, interference and diffraction of light. 1980: Elsevier.

34. Miyazaki, H., et al., Thermal emission of two-color polarized infrared waves from integrated plasmon cavities. Applied Physics Letters, 2008. 92(14): p. 141114.

35. Yokoyama, T., et al., Spectrally Selective Mid-Infrared Thermal Emission from Molybdenum Plasmonic Metamaterial Operated up to $1000^{\circ}$ C. Advanced Optical Materials, 2016. 4(12): p. 1987-1992.

36. Chirumamilla, M., et al., Multilayer tungsten-alumina-based broadband light absorbers for high-temperature applications. Optical Materials Express, 2016. 6(8): p. 2704-2714.

37. Dean, J., Lange's handbook of chemistry. Material and manufacturing process, 1990. 5(4): p. $687-688$. 